\title{
Six essential conditions for bladder-sparing strategies in bacillus Calmette-Guérin unresponsive bladder cancer
}

\author{
Shahrokh F Shariat*1,2,3,4,5, Dmitry V Enikeev ${ }^{5}$ \& Hadi Mostafaei ${ }^{1,6}$ \\ ${ }^{1}$ Department of Urology, Comprehensive Cancer Center, Medical University of Vienna, 1090, Vienna, Austria \\ ${ }^{2}$ Department of Urology, Weill Cornell Medical College, New York, NY 10065, USA \\ ${ }^{3}$ Department of Urology, University of Texas Southwestern, Dallas, TX 75390, USA \\ ${ }^{4}$ Department of Urology, Second Faculty of Medicine, Charles University, 15006, Prague, Czech Republic \\ ${ }^{5}$ Institute for Urology \& Reproductive Health, I.M. Sechenov First Moscow State Medical University, 119048, Moscow, Russia \\ ${ }^{6}$ Research Center for Evidence-Based Medicine, Tabriz University of Medical Sciences, 5166, Tabriz, Iran \\ *Author for correspondence: Tel.: +43 140400 26150; Fax: +43 140400 2332; shahrokh.shariat@meduniwien.ac.at
}

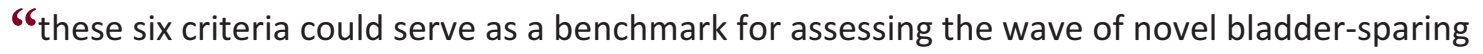
therapies that are entering the BCG-unresponsive NMIBC space. It suffices if they are easier and better tolerated than radical cystectomy, while not resulting in worse oncologic outcomes."
\end{abstract}

First draft submitted: 22 May 2019; Accepted for publication: 10 July 2019; Published online: 6 August 2019

Keywords: bacillus Calmette-Guérin $\bullet$ BCG unresponsive $\bullet$ bladder cancer $\bullet$ immunotherapy $\bullet$ non muscle invasive bladder cancer $\bullet$ salvage therapies

Bladder cancer is the sixth most common cancer in the USA, with 80,470 estimated new cases in 2019 and 17,670 estimated deaths. In developed countries, the majority of patients are diagnosed with nonmuscle invasive bladder cancer (NMIBC; i.e., tumor limited to mucosa and submucosa but not invading the muscularis propria layer). The large majority of NMIBC patients have an excellent survival, but significant subgroups experience disease recurrence and/or progression to muscle-invasive bladder cancer (MIBC). To deliver the best therapy for each patient, NMIBC patients are categorized into low-, intermediate- and high-risk groups based on clinical and pathological characteristics [1,2]. Since patients with high-risk NMIBC (i.e., patients with carcinoma in situ [CIS], high-grade $\mathrm{T} 1$ or high-grade $\mathrm{Ta}$ ) have the highest probabilities of disease recurrence and progression, they are considered for a 3-year course of adjuvant induction plus a maintenance course of intravesical bacillus Calmette-Guérin (BCG) immunotherapy [2].

BCG immunotherapy has been effectively used in the last 30 years for the management of high-risk NMIBC [3]. However, despite clinical familiarity with BCG, its molecular mode of action remains only partially understood. Both benign and cancerous urothelial cells, together with the local immune system, play an active role in BCG's therapeutic anti-tumor effect. These cells are involved in the attachment and internalization of BCG, which leads to the activation and secretion of cytokines and chemokines. BCG is then presented to immune cells including $\mathrm{CD}^{+}$and $\mathrm{CD}^{+}$lymphocytes, natural killer cells, granulocytes, macrophages and dendritic cells. The cancer cells are then eventually killed through direct and indirect cytotoxic effects. Secretion of soluble factors such as tumor necrosis factor-related apoptosis-inducing ligand and a limited direct effect also contribute to BCG's effect [4-7].

While the majority of patients initially respond to BCG therapy, most eventually experience disease recurrence and a smaller proportion suffer from disease progression to MIBC [8]. To date, there is no effective and accepted salvage noninvasive therapy for patients who do not respond to BCG therapy [9]. The current standard of care for these patients is radical cystectomy with a urinary diversion. Radical cystectomy is, however, associated with significant morbidity and mortality [10,11]. In addition, it could be considered as overtreatment for many patients with BCGunresponsive NMIBC. Development and discovery of treatment options for patients with BCG-unresponsive NMIBC is, therefore, of major importance to patients and their physicians in order to curb unnecessary treatmentrelated morbidity, healthcare expenditures and mortality. 
Recognizing this unmet need and the challenges associated with radical cystectomy in patients with BCGunresponsive NMIBC, the US FDA has stated that "single-arm trials are appropriate in clinical settings where a randomized controlled trial is either unethical or not feasible" [17]. This has ushered in a number of singlearm Phase I/II clinical trials, enrolling patients who either are not fit for or refuse radical cystectomy in this disease space. There has also been special enthusiasm for immune checkpoint inhibitors, since bladder cancer is an immune-responsive disease and drugs targeting immune checkpoints have shown significant benefit in advanced and metastatic urothelial carcinoma [12].

To guide the trial designs, here we discuss the prerequisite criteria required for a salvage bladder-sparing intervention to be considered as an adequate alternative to radical cystectomy in patients with BCG-unresponsive NMIBC. It is obvious that the equivalence to radical cystectomy, the standard of care in this disease space, can only be assessed in a prospective randomized controlled clinical trial. Since such a study is nearly impossible due to the patient and physician lack of enthusiasm, we have attempted to define criteria that have to be fulfilled for a salvage bladder-sparing intervention to gain assent.

\section{Inclusion of only patients with BCG-unresponsive NMIBC}

Various differing definitions of BCG failure have been put forth over the years. Recently, separate consensus panels have described the defining characteristics of patients who are not likely to benefit from additional BCG and should, therefore, be considered for a change of therapy. The intention of these definitions is to avoid patients receiving treatments from which they are unlikely to benefit, as well as to aid in future clinical trial designs by establishing appropriate eligibility criteria for studies of novel therapies for patients with persistent or recurrent tumors despite BCG treatment.

First, it is agreed upon that BCG intolerance (i.e., did not fail BCG but did not tolerate BCG) is not considered as a part of BCG unresponsive. For a patient to be categorized as BCG unresponsive, he or she needs to have received adequate BCG.

The US FDA defines BCG unresponsive as "persistent high-grade disease or recurrence within 6 months of receiving at least two courses of intravesical BCG (at least five of six induction doses and at least two of three maintenance doses); or T1 high-grade disease at the first evaluation following induction BCG alone (at least five of six induction doses)" [13].

The European Association of Urology NMIBC guideline group and the International Bladder Cancer Collaboration Group (IBCG) have defined BCG unresponsive as, patients who have BCG-refractory disease and those who experience BCG relapse within 3 or 6 months of last BCG therapy, respectively. All agree on the condition that patients must have received adequate prior BCG therapy prior to be defined as unresponsive. A 6-week BCG induction plus a minimum of 1-and 3-week maintenance should occur at the 6 months period after diagnosis of high-risk NMIBC. BCG refractory is defined as persistent high-grade disease at 6 months despite adequate BCG treatment. This includes any stage/grade progression at 3 months. Specifically, CIS at 3 months is not considered as refractory, but persistence of CIS at 6 months is. BCG relapsing is defined as recurrence of high-grade disease after achieving a disease-free state at 6 months milestone after adequate BCG treatment [14,15].

\section{The salvage therapy should be easier \& cheaper to perform than radical cystectomy}

Radical cystectomy is a highly complex surgical intervention that requires a significant teamwork and individual learning curve. The alternative to radical cystectomy should be less difficult in execution and, ideally, more reproducible. This criteria is complied by almost all salvage interventions currently being tested in the BCG unresponsive space.

\section{The salvage therapy should be safer than radical cystectomy}

Another shortcoming of radical cystectomy with urinary diversion is its associated morbidity and mortality. In a retrospective review at a high volume expert center, Shabsigh et al. reported complications in 64\% (735 of 1142) of patients within 90 days of surgery [16]. The 30 -day mortality rate was $1.5 \%$. For a salvage intervention to replace radical cystectomy, it needs to be less morbid. This criteria is fulfilled by almost all salvage interventions currently being tested in the BCG unresponsive space. 


\section{The efficacy of the salvage intervention should be clinically significant \& durable}

The primary efficacy end point in single-arm trials of patients with BCG-unresponsive NMIBC should be the complete response rate in high-risk patients. It is also very important to consider the duration of response, and therefore there should be a minimum follow-up of at least 12 months. Partial response is very difficult and probably meaningless in this disease setting. Complete response should be defined as absence of any evidence of disease including a negative cystoscopy and a negative urine cytology. The appearance of a low-grade papillary lesion after salvage therapy is considered as a nonevent (cytology being negative).

A consistently difficult question is the disease status in the upper urinary tract and prostatic urethra. If the investigated salvage treatment is delivered, aiming to treat only intravesically, then the development of disease in the upper urinary tract and/or the prostatic urethra cannot be attributed to a lack of activity of the investigated drug. Thus, sponsors can consider patients with new malignant lesions of the upper tract or prostatic urethra but no signs of disease in the bladder, who have received salvage intravesical therapy, 'have achieved a complete response' in the primary analysis. Systemic therapies, in contrast, are expected to have a treatment effect throughout the urinary tract. Therefore, a patient who received systemic therapy cannot be considered to have a complete response if the patient has a malignant lesion(s) in the upper tract or prostatic urethra.

One question that has consistently been brought up is whether a delay in radical cystectomy can be a trial end point. Despite being a direct patient benefit, the variations in patient and healthcare provider preferences can confound the interpretation of this end point in randomized trials and particularly in single-arm trials. Therefore, this information should be collected, yet it should not be the primary end point in any trial.

\section{The risk of missing 'window of opportunity' for radical cystectomy should be minimal to none} The reason to perform 'bladder-sparing' intervention in patients with BCG-unresponsive NMIBC is to avoid radical cystectomy. While even a delay to radical cystectomy could be considered as a benefit, it is a difficult end point to measure reliably in a single arm trial (see above). On the other hand, disease progression to muscle-invasive and/or metastatic disease is a compelling significant end point. While some patients may even initially respond to the bladder-sparing intervention, they may eventually experience disease recurrence triggering a radical cystectomy before they experience disease progression to MIBC. In other words, an attempt at sparing the bladder should not result in a high rate of patients losing 'window of opportunity' for the local therapy with curative intent that is radical cystectomy. Patients with NMIBC who experience disease progression to MIBC have, indeed, a significantly worse survival than those present with primary MIBC (i.e., without a history of NMIBC) [17].

\section{The salvage intervention should not make the complications of potential radical cystectomy prohibitive}

It is also important that for the patients who eventually fail the bladder-sparing therapy, a timely radical cystectomy can be performed without a disproportional increase in morbidity (e.g., intraoperative complications, loss of the possibility of performing a nerve-sparing radical cystectomy with a neobladder). For standard bladder preserving strategies in MIBC disease space, this is not always the case. For example, trimodal therapy has been advocated for selected patients with small volume organ confined MIBC [18]. While this therapy may not confer a worse survival, 30-60\% of the patients eventually need a salvage radical cystectomy for disease recurrence [19]. The surgery in the post-radiation therapy is more morbid (i.e., higher rate of complications), and the patient does not have the opportunity of receiving a nerve-sparing radical cystectomy or a neobladder anymore, for which he would have been ideal candidate in the first place [20]. This is because of the effect of the radiotherapy on the tissues. In other words, for a bladder-sparing therapy to take foothold in the BCG unresponsive space, it needs not only to be reproducible, safe and effective, but it should also not foreclose the opportunity for a high quality radical cystectomy, when necessary.

In conclusion, these six criteria could serve as a benchmark for assessing the wave of novel bladder-sparing therapies that are entering the BCG-unresponsive NMIBC space. It suffices if they are easier and better tolerated than radical cystectomy, while not resulting in worse oncologic outcomes. They should not lead to delay of radical cystectomy (window of opportunity) if needed and they should not make the radical cystectomy, again, if needed, more complicated or morbid. The last criteria to consider is also the financial cost-effectiveness in the context of a value-based approach to healthcare. 


\section{Financial \& competing interests disclosure}

SF Shariat receives honoraria's from Astellas. Astra Zeneca, Bayer, BMS, Cepheid, Ferring, Ipsen, Janssen, Lilly, MSD, Olympus, Pfizer, Pierre Fabre, Richard Wolf, Roche, Sanochemia, Sanofi, Takeda, Urogen. He also has a consulting and advisory role at these companies. The authors have no other relevant affiliations or financial involvement with any organization or entity with a financial interest in or financial conflict with the subject matter or materials discussed in the manuscript apart from those disclosed.

No writing assistance was utilized in the production of this manuscript.

\section{References}

1. Soukup V, Čapoun O, Cohen D et al. Risk stratification tools and prognostic models in non-muscle-invasive bladder cancer: a critical assessment from the European Association of Urology Non-muscle-invasive Bladder Cancer Guidelines Panel. Eur.Urol. Focus In Press (2018).

2. Babjuk M, Böhle A, Burger M et al. EAU guidelines on non-muscle-invasive urothelial carcinoma of the bladder: update 2016. Eur. Urol. 71(3), 447-461 (2017).

3. D'Andrea D, Gontero P, Shariat SF, Soria F. Intravesical bacillus Calmette-Guérin for bladder cancer: are all the strains equal? Transl. Androl. Urol. 8(1), 85 (2019).

4. Ratliff TL, Palmer JO, McGarr JA, Brown EJ. Intravesical Bacillus Calmette-Guerin therapy for murine bladder tumors: initiation of the response by fibronectin-mediated attachment of Bacillus Calmette-Guerin. Cancer Res. 47(7), 1762-1766 (1987).

5. BÖHLE A, Brandau S. Immune mechanisms in bacillus Calmette-Guerin immunotherapy for superficial bladder cancer. J. Urol. 170(3), 964-969 (2003).

6. Luo Y, Chen X, O'donnell MA. Role of Th1 and Th2 cytokines in BCG-induced IFN- $\gamma$ production: cytokine promotion and simulation of BCG effect. Cytokine 21(1), 17-26 (2003).

7. Zuiverloon TC, Nieuweboer AJ, Vékony H, Kirkels WJ, Bangma CH, Zwarthoff EC. Markers predicting response to bacillus Calmette-Guerin immunotherapy in high-risk bladder cancer patients: a systematic review. Eur. Urol. 61(1), 128-145 (2012).

8. Gontero P, Sylvester R, Pisano F et al. Prognostic factors and risk groups in T1G3 non-muscle-invasive bladder cancer patients initially treated with bacillus Calmette-Guérin: results of a retrospective multicenter study of 2451 patients. Eur. Urol. 67(1), 74-82 (2015).

9. Hassler MR, Shariat SF, Soria F. Salvage therapeutic strategies for bacillus Calmette-Guerin failure. Curr. Opin. Urol. 29(3), 239-246 (2019).

10. Grubmueller B, Seitz C, Shariat SF. The treatment of muscle-invasive bladder cancer in geriatric patients. Curr. Opin. Urol. 26(2), 160-164 (2016).

11. Lee RK, Abol-Enein H, Artibani W et al. Urinary diversion after radical cystectomy for bladder cancer: options, patient selection, and outcomes. BJU Int. 113(1), 11-23 (2014).

12. Resch I, Shariat SF, Gust KM. PD-1 and PD-L1 inhibitors after platinum-based chemotherapy or in first-line therapy in cisplatin-ineligible patients. Memo 11(1), 43-46 (2018).

13. FDA. BCG-unresponsive nonmuscle invasive bladder cancer: developing drugs and biologics for treatment guidance for industry. (2018). https://www.fda.gov/media/101468/download

14. Shore ND, Boorjian SA, Canter DJ et al. Intravesical rAd-IFN $\alpha /$ Syn3 for patients with high-grade, bacillus calmette-guerin-refractory or relapsed non-muscle-invasive bladder cancer: a Phase II randomized study. J. Clin. Oncol. 35(30), 3410 (2017).

15. Kamat AM, Sylvester RJ, Böhle A et al. Definitions, end points, and clinical trial designs for non-muscle-invasive bladder cancer: recommendations from the International Bladder Cancer Group. J. Clin. Oncol. 34(16), 1935 (2016).

16. Shabsigh A, Korets R, Vora KC et al. Defining early morbidity of radical cystectomy for patients with bladder cancer using a standardized reporting methodology. Eur. Urol. 55(1), 164-176 (2009).

17. van den Bosch S, Alfred Witjes J. Long-term cancer-specific survival in patients with high-risk, non-muscle-invasive bladder cancer and tumour progression: a systematic review. Eur. Urol. 60(3), 493-500 (2011).

18. Ploussard G, Daneshmand S, Efstathiou JA et al. Critical analysis of bladder sparing with trimodal therapy in muscle-invasive bladder cancer: a systematic review. Eur. Urol. 66(1), 120-137 (2014).

19. Mathieu R, Lucca I, Klatte T, Babjuk M, Shariat SF. Trimodal therapy for invasive bladder cancer: is it really equal to radical cystectomy? Curr. Opin. Urol. 25(5), 476-82 (2015).

20. Ploussard G, Daneshmand S, Efstathiou JA, Herr HW et al. Critical analysis of bladder sparing with trimodal therapy in muscle-invasive bladder cancer: a systematic review. Eur. Urol. 66(1), 120-137 (2014). 